\title{
Vértigo posicional paroxístico benigno: Factores de riesgo asociados y eficacia de las maniobras de reposición
}

\author{
Benign paroxysmal positional vertigo: Associated risk factors and efficacy \\ of particle repositioning maneuvers
}

\author{
Phoebe Ramos Y1, Sofía Waissbluth A', Diego Correa F1, Karina Aracena C1.
}

\begin{abstract}
RESUMEN
Introducción: El vértigo posicional paroxístico benigno (VPPB) es el vértigo periférico más frecuente. El tratamiento depende del compromiso de los canales semicirculares (CSC) y/o cúpulas, y consiste en maniobras de reposición de partículas.

objetivo: Evaluar los factores de riesgo asociados al VPPB en pacientes atendidos en el Servicio de Otorrinolaringología de la Red de Salud UC Christus. Evaluar la tasa de éxito de las maniobras de reposición.

Material y método: Estudio retrospectivo. Se revisaron casos de VPPB con indicación de maniobras de reposición durante los años 2016-2017. Se obtuvo información demográfica, antecedentes médicos, la maniobra realizada y su éxito. Se evaluaron comorbilidades y temporada del año.

Resultados: Se incluyeron 195 consultas, realizándose 293 maniobras. La mayoría de los pacientes fueron mujeres (74\%) con edad promedio de 63 años. Comorbilidades más frecuentes fueron hipertensión, dislipidemia y diabetes mellitus. El $20 \%$ presentó una hipofunción vestibular concomitante, 23\% presentó antecedentes de VPPB y 8\% compromiso bilateral. Canalolitiasis del CSC posterior fue predominante (90\%). En el $77,3 \%$ se resuelve el caso con una maniobra. Los casos fueron más frecuentes en primavera y otoño.

Conclusiones: EI VPPB fue más frecuente en mujeres, con una edad promedio de 63 años. La mayoría presentó canalolitiasis unilateral lográndose resolución con una maniobra de reposición.
\end{abstract}

Palabras clave: Vértigo periférico, posicional, paroxístico, benigno, maniobras.

\begin{abstract}
Introduction: Benign paroxysmal positional vertigo (BPPV) is the most common cause of peripheral vertigo. The treatment depends on the semicircular canal (SCC) and/ or cupula involved and consists of particle repositioning maneuvers.
\end{abstract}

1 Departamento de Otorrinolaringología, Pontificia Universidad Católica de Chile, Santiago, Chile.

Los autores declaran no tener conflictos de interés.

Recibido el 17 de agosto de 2019. Aceptado el 21 de noviembre de 2019. 
Aim: Analyze risk factors associated with BPPV for patients seen at the otorhinolaryngology department of the UC Christus health center. Evaluate the success rate of the repositioning maneuvers.

Material and methods: Retrospective study. All cases of BPPV for which a repositioning maneuver was prescribed during the years 2016-2017 were reviewed. Data obtained includes demographics, medical history, maneuver performed, and its success rate. Comorbidities and seasonality were evaluated.

Results: 195 cases were included; with 293 maneuvers. The majority were women (74\%), and the average age was 63 years. Common comorbidities were hypertension, dyslipidemia and diabetes mellitus. Concurrently, 20\% had unilateral vestibular hypofunction, $23 \%$ had a history of BPPV, and $8 \%$ had bilateral involvement. Posterior SCC canalithiasis was most common (90\%). In $77.3 \%$, the case was resolved with one maneuver. Cases were most frequent in the spring and autumn season.

Conclusion: BPPV was more common in women with an average age of 63 years. The majority of patients presented with unilateral canalithiasis obtaining a complete recovery with a single maneuver.

Key words: Peripheral vertigo, positional, paroxysmal, benign, maneuvers.

\section{INTRODUCCIÓN}

El vértigo posicional paroxístico benigno (VPPB) se considera el vértigo periférico más frecuente representando aproximadamente $25 \%$ de todos los vértigos de origen vestibular ${ }^{1-4}$. En la literatura, se estima una prevalencia durante la vida de $2,4 \%$ de la población ${ }^{2,5}$. Generalmente se presenta en pacientes de mayor edad, entre las edades de 45 a 70 años, incluso se ha descrito que pacientes mayores de 70 años poseen $30 \%$ de probabilidades de tener al menos un episodio en su vida ${ }^{2,6-8}$. Presenta mayor incidencia en el sexo femenino en proporción de 2:12,7. Es un síndrome vestibular episódico paroxístico que se describe como un trastorno otoconial causado por la degeneración y movilización de las otoconias que se desprenden de la membrana otolítica del utrículo desplazándose hacia los CSC9. Los cambios de posición de la cabeza hacen que estos otolitos u otoconias, viajen dentro del CSC lo que provoca movimiento de la endolinfa causando la sensación de vértigo y nistagmo característico $0^{4,9}$. Clínicamente se caracteriza por episodios recurrentes autolimitados de vértigo rotatorio desencadenados por cambios de posición de la cabeza en el espacio. Suele asociarse a náuseas e incluso vómitos, a diferencia de otros síndromes no se presenta con síntomas auditivos ni neurológicos ${ }^{1,2}$. Para el diagnóstico se deben realizar maniobras específicas de provocación que desencadenan un nistagmo característico cuya dirección nos dará el diagnóstico de localización específica². El CSC posterior es el que con mayor frecuencia se afecta en $60 \%-90 \%$, seguido por el compromiso de CSC horizontal 10\%-25\%; la afectación del CSC superior es la menos frecuente $1 \%-2 \% 2,5$. Existen diversas maniobras de provocación que gatillarán signología específica según el canal CSC comprometido.

La etiología exacta de esta patología aún se desconoce, sin embargo, se clasifican en VPPB primario o idiopático (más del $50 \%$ de los casos) y secundario (i.e. traumatismo encéfalo craneano, patología de oído interno como laberintitis viral 0 hidropesía endolinfática, otitis media crónica, otoesclerosis, cirugía de oído, cirugías prolongadas o reposo en cama prolongado, entre otros) $)^{2,5,10}$. En la literatura se describe asociación de VPPB a diversas patologías como, por ejemplo: osteoporosis, déficit de vitamina $D$, patologías cardiovasculares, patología psiquiátrica, otras enfermedades auditivas (i.e. enfermedad de Ménière), alergia y trastornos inmunológi$\cos ^{1-3,11}$.

El tratamiento depende del CSC comprometido y consiste en maniobras de reposición de partículas que son el tratamiento de primera línea, y frecuentemente son curativas ${ }^{7}$. La tasa de éxito depende del CSC comprometido y la maniobra 
realizada. En la literatura se describe que en pacientes con compromiso del CSC posterior la maniobra de Epley posee una tasa de resolución de aproximadamente $75 \%$, siendo esta maniobra muy efectiva2,12. En comparación, los pacientes que presentan compromiso de los CSC laterales suelen presentar tasas de éxito variables, según adonde está localizado el compromiso (cupulolitiasis versus canalolitiasis) y según la maniobra realizada. Es frecuente observar la necesidad de realizar más de una maniobra para este $\operatorname{CSC}^{13,14}$. Carnevale y cols describen una tasa de éxito de $50 \%$ y $75 \%$ para VPPB del CSC lateral por cupulolitiasis y canalolitiasis, respectivamente ${ }^{2}$. A pesar del tratamiento efectivo para el VPPB, estos episodios tienden a recurrir, y se estima que existe $44 \%$ de recidiva durante los primeros dos años, y $50 \%$ en cinco años $7,9,15$. Pueden recurrir en un conducto diferente $\mathrm{y} / 0$ en el oído contralateral al primer episodio de vértigo.

\section{OBJETIVO}

Los objetivos de este estudio son evaluar los factores de riesgo asociados al VPPB en pacientes atendidos en el Servicio de Otorrinolaringología de la Red de Salud UC Christus, y evaluar la tasa de éxito de las maniobras de reposición.

\section{MATERIAL Y MÉTODO}

Se realizó un estudio retrospectivo, descriptivo, de todos los pacientes con indicación de maniobras de reposición por VPPB durante los años 2016 y 2017 en el Servicio de Otorrinolaringología de la Red de Salud UC Christus. La búsqueda reveló 195 consultas de VPPB, pacientes quienes fueron diagnosticados con VPPB según los criterios diagnósticos de la Sociedad de Bárány ${ }^{16}$, con un total de 293 maniobras realizadas. Se revisaron datos epidemiológicos, antecedentes médicos, la maniobra de reposición realizada, y su tasa de éxito. Se evaluó además la temporada del año durante la cual los pacientes consultaron. El estudio cuenta con la aprobación del Comité de Ética de la Facultad de Medicina de la Pontificia Universidad Católica de Chile.

\section{RESULTADOS}

\section{Demográfica}

La serie está constituida por 192 pacientes, con una edad promedio de 63 años (rango 15-95 años); 142 mujeres (74\%) y 50 hombres. Tres pacientes consultaron más de una vez por cuadros recurrentes durante los dos años, con un total de 195 consultas. Solo 9 casos $(4,7 \%)$ fueron VPPB secundarios a un traumatismo encefalocraneano; no se describen casos secundarios a otras causas. Sin embargo, 14 pacientes (7,3\%) presentaron el antecedente de osteopenia u osteoporosis, y 39 pacientes $(20,3 \%)$ presentaron paresia vestibular concomitante. De éstos, 29 pacientes $(74,4 \%)$ con compromiso ipsilateral al oído comprometido por VPPB, aunque clínicamente no se describen rotundamente síntomas de hipofunción vestibular que lleve a un diagnóstico preciso de VPPB secundario.

\section{Comorbilidades}

Las comorbilidades más frecuentes fueron hipertensión arterial (45\%), dislipidemia (30\%), diabetes 0 resistencia a la insulina (22\%), hipotiroidismo $(20 \%)$ y trastornos psiquiátricos $(20 \%)$. En este último grupo, fueron dos veces más frecuentes los trastornos del estado de ánimo con respecto a los trastornos de ansiedad. Veinticuatro por ciento de los pacientes presentó el antecedente de algún trastorno inmunológico siendo lo más frecuente las alergias a los medicamentos y la rinitis alérgica (Figura 1).

Se observó que $17 \%$ de los pacientes estaban siendo suplementados con calcio $y / 0$ vitamina $D$ ya sea por el diagnóstico de osteopenia u osteoporosis, 0 considerando niveles de vitamina $D$ menores a $30 \mathrm{mg} / \mathrm{dl}$. En cuanto a los antecedentes en relación a la función vestibular, se observó al menos una alteración en $44 \%$ de los pacientes ( $n=85$ ), y de ellos, $20 \%$ presentó más de una alteración. El antecedente más importante fue haber presentado un cuadro de VPPB previamente (23\%). El 20\% presentó una hipofunción vestibular concomitante al cuadro de VPPB, cuantificando el reflejo vestíbulo-ocular con prueba calórica; y en el $74 \%$ de los casos fue ipsilateral. Siete pacientes 


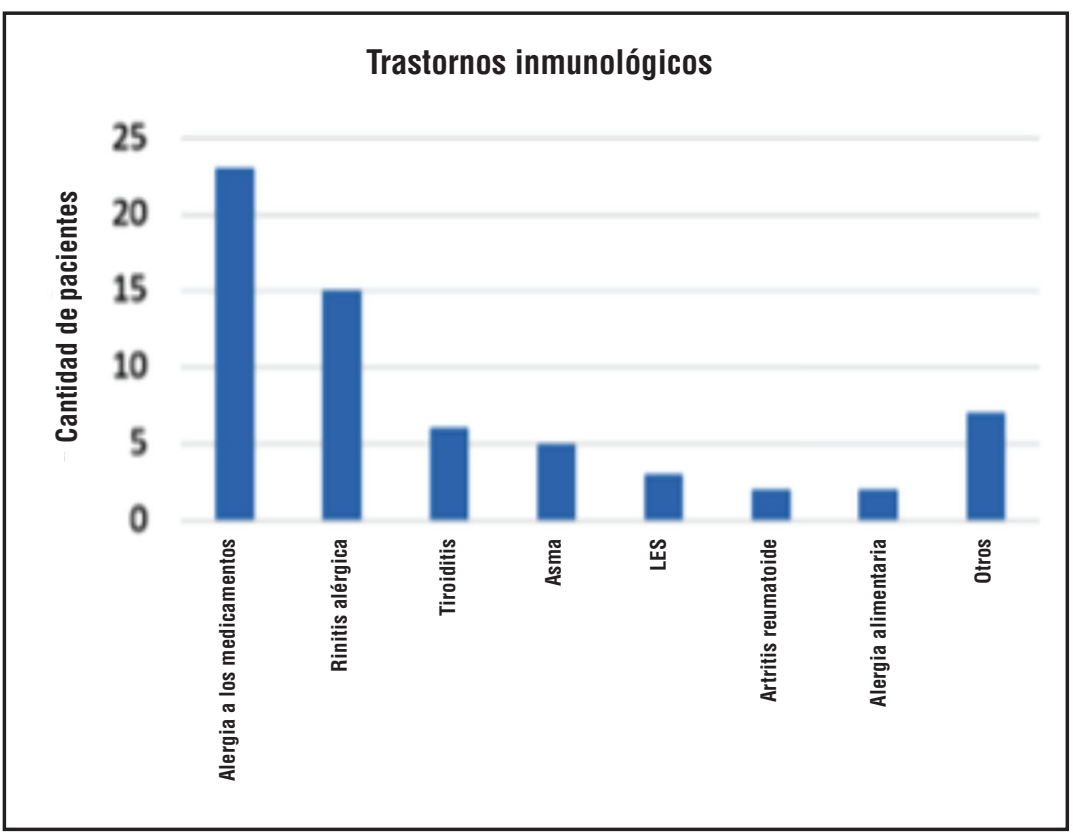

Figura 1. Observación de trastornos inmunológicos en los pacientes que consultaron por VPPB. Otros: hepatitis $(n=1)$, síndrome de Sjögren ( $n=1)$, vitíligo $(n=1)$, uveítis $(n=1)$, vasculitis $(n=1)$, psoriasis $(n=1)$, dermatitis de contacto $(n=1)$.

refieren un cuadro de paresia cocleovestibular 0 hipoacusia súbita anteriormente, 9 refieren enfermedad de Ménière (5 casos son ipsilaterales), 2 refieren otosclerosis, y ninguno refiere migraña vestibular. No se describe ningún caso de vértigo posicional central.

\section{Maniobras de reposición}

Hubo 195 consultas para realizar maniobras de reposición para 192 pacientes durante los dos años evaluados. De ellos, 16 pacientes presentaron compromiso bilateral (compromiso del lado derecho en $n=115$, y compromiso del lado izquierdo $n=96$ ). En 4 casos, al iniciar la evaluación premaniobra, se evidencia resolución espontánea del cuadro sin necesidad de realizar la maniobra de reposición. La canalolitiasis consiste en el compromiso más prevalente, evidenciándose en el $97 \%$ de las consultas, siendo predominante el compromiso del CSC posterior $(89,6 \%)$ en $55 \%$ de los casos del oído derecho, seguido del compromiso del CSC lateral $(9,4 \%)$, y finalmente el CSC superior $(0,9 \%)$. En 7 casos, hubo compromiso de más de un CSC de forma ipsilateral, evidenciándose durante los controles de las maniobras de reposición. En 15 casos, hubo cupulolitiasis (determinado por la presencia de nistagmo posicional obtenido tras una breve latencia 0 sin latencia mediante la maniobra de rotación cefálica correspondiente según el canal estimulado y prolongándose por más de un minuto) ${ }^{16}$ con 0 sin compromiso de un CSC siendo la cúpula posterior $(n=8)$ y lateral $(\mathrm{n}=7)$ afectadas de forma similar. En cuanto a las maniobras de reposición, en el $77,3 \%$ se resuelve el caso de VPPB con una sola maniobra. En 4 casos, se requirió $\geq 5$ maniobras (Tabla 1 ). En total, se realizaron 293 maniobras. En los casos en los que fue necesario realizar $\geq 3$ maniobras ( $n$ $=17$ ), fue frecuente evidenciar canalolitiasis junto a cupulolitiasis (Figura 2).

De estos pacientes, 9 presentaron cupulolitiasis (6/9 cúpula lateral, $3 / 9$ cúpula posterior). Al comparar cada maniobra por sí sola, la maniobra de Epley para el CSC posterior obtuvo una tasa de éxito de $76 \%$ con la realización de una sola maniobra con $23 \%$ de los pacientes requiriendo más de una maniobra. La maniobra de Epley 
Tabla 1. Cantidad de maniobras realizadas en total, y durante cada consulta

\begin{tabular}{|lrc|}
\hline Cantidad de maniobras & $n$ & \% maniobras realizadas por consulta \\
\hline 0 maniobras & 4 & - \\
1 maniobra & 160 & 77,3 \\
2 maniobras & 30 & 14,5 \\
3 maniobras & 6 & 2,9 \\
4 maniobras & 7 & 3,4 \\
$\geq 5$ maniobras & 4 & 1,9 \\
\hline
\end{tabular}

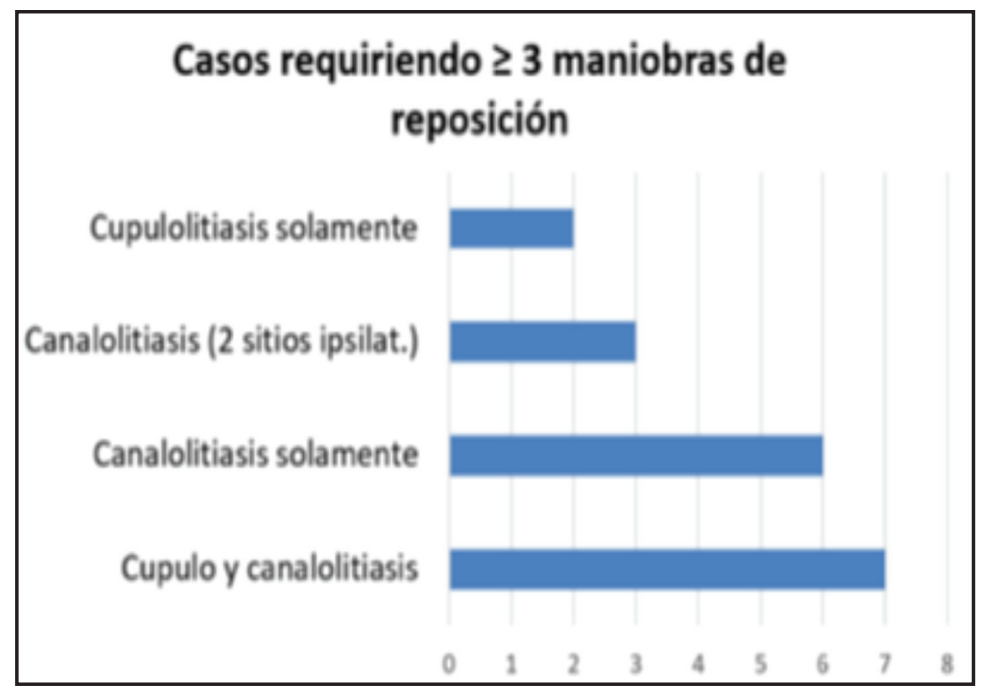

Figura 2. Sitios vestibulares comprometidos para los pacientes presentando con VPPB que requiere 3 o más maniobras de reposición para la resolución del cuadro. *Un paciente presentó canalolitiasis de 2 sitios ipsilaterales además de cupulolitiasis.

modificada para el CSC posterior obtuvo una tasa de éxito de 95\% con una sola maniobra. Esta maniobra difiere en que consiste en realizar la maniobra de Epley pero con un mayor número de movimientos y pasos (1. se lleva al paciente de la posición sentada a decúbito prono con la cabeza colgando girada hacia el lado afectado en $45^{\circ}, 2$. se gira la cabeza en $30^{\circ}$ hacia el lado opuesto, 3. se gira la cabeza $15^{\circ}$ llegando a la posición de cabeza colgando en decúbito dorsal a $0^{\circ}$, 4. girar la cabeza $15^{\circ}$ hacia el lado sano, 5. girar en $30^{\circ}$ quedando en posición $45^{\circ}$ hacia el lado sano 6 . posteriormente se gira la cabeza en $90^{\circ}$ hacia el lado sano queda esta inclinada diagonalmente hacia abajo (posición decúbito ventral), 7. por último, se gira la cabeza $45^{\circ}$ quedando en decúbito ventral mirando al suelo, se esperan 5 minutos en esta posición y se completa la maniobra -5 minutos entre cada posición ${ }^{17}$.

Para el compromiso del CSC lateral, las maniobras más frecuentemente usadas fueron las maniobras de Lempert $(80 \%)$, Semont $(20 \%)$ y Gufoni $(20 \%)$. Los porcentajes están en relación al total de los pacientes con compromiso del CSC lateral, se sobreponen ya que en el $56 \%$ de los pacientes se requirió más de una maniobra para este CSC (2 maniobras: $24 \%, 3$ maniobras: $8 \%, \geq 4$ maniobras: $24 \%$ ). La maniobra de Lempert para el CSC lateral obtuvo una tasa similar a la de Epley para el CSC posterior, con un éxito de $76 \%$ aplicando una sola maniobra, con $23 \%$ de los pacientes requiriendo más de una. 
En cuanto a la localización específica, los pacientes con canalolitiasis del CSC posterior, $78 \%$ requirió de solo una maniobra para su tratamiento, en cambio los pacientes con canalolitiasis del CSC lateral, $68 \%$ requirió una maniobra para su tratamiento, cabe destacar que existía un mayor número de maniobras en este grupo para lograr remisión de la sintomatología al igual que los pacientes que presentaban cupulolitiasis. Se evaluó igualmente durante qué período del año los pacientes consultaban por cuadros de VPPB. Los casos fueron más frecuentes en la primavera (28\%), seguido de la temporada de otoño $(27 \%)$.

\section{DISCUSIÓN}

La prevalencia de mareos y vértigo en la población general es de aproximadamente $20 \%-30 \%$ siendo el VPPB la forma más frecuente de vértigo periférico, sobre todo en edades entre 50-70 años, lo que es concordante con los resultados de este estudio4. Se debe considerar que esta patología puede tener una asociación significativa con diversas comorbilidades. En este estudio predominaron las comorbilidades cardiovasculares (hipertensión arterial, dislipidemia, diabetes mellitus o resistencia a la insulina), comorbilidades que, si bien se asocian a mayor riesgo de VPPB según lo descrito en la literatura, debemos considerar que, en la población chilena, este conjunto de patologías es frecuente, por lo que no se puede establecer una correlación entre éstas y el VPPB con los resultados de este tipo de estudio 2,8,18.

Por otro lado, en relación a los trastornos psiquiátricos, $20 \%$ de los pacientes de este estudio presentaron el antecedente de trastorno del estado del ánimo lo que es concordante con la literatura'. Los diagnósticos más frecuentemente asociados al VPPB son la depresión, ansiedad generalizada y trastornos de personalidad (trastorno obsesivo compulsivo); al mismo tiempo el VPPB puede desencadenar sintomatología psiquiátrica en pacientes sin antecedentes dado que la mayoría de las veces termina restringiendo actividades diarias con consecuencias psicosociales ${ }^{1}$.

Además, se observó en $17 \%$ de los pacientes la necesidad de suplementación con calcio y/o vitamina $\mathrm{D}$, lo que se relaciona con la asociación del VPPB a osteoporosis y osteopenia ya que estudios basados en densitometrías óseas y los niveles serológicos de la vitamina $D$ describen que pacientes con déficit de vitamina $D$ presentan mayor riesgo de desarrollar VPPB y también se asociaría a mayor recurrencia ${ }^{3}$. Las otoconias están formadas por carbonato de calcio y por lo tanto el metabolismo del calcio es de gran interés para esta patología. Para la síntesis de vitamina $D$ se requiere exposición al sol (rayos UVB), por lo que es de esperar que en temporadas más frías y oscuras con menor exposición a la luz solar es posible que exista menor síntesis de vitamina $D$ y consecuentemente mayor riesgo de $\mathrm{VPPB}^{19}$. En nuestro estudio, los pacientes presentaban VPPB más frecuentemente en primavera seguido de la temporada de otoño distinto a lo descrito en la literatura, donde los casos de VPPB se presentan con mayor frecuencia en otoño e invierno ${ }^{19}$. Esto podría deberse a que desconocemos el tiempo ocurrido entre el inicio del síntoma y el diagnóstico propiamente tal.

En relación a la función vestibular, 20\% presentaba una hipofunción vestibular asociada al VPPB, un porcentaje menor presentó enfermedad de Ménière concomitante, situación descrita en la literatura ${ }^{20}$. Los pacientes con enfermedad de Ménière presentan mayor riesgo de desarrollar VPPB con una incidencia variable entre $0,5 \%$ a $30 \%$, y que éste compromete con mayor frecuencia los CSC laterales siendo de difícil manejo. Se cree que el hidrops endolinfático podría generar un desprendimiento de los otolitos 0 a través de cambios estructurales en el acueducto vestibular gatillaría el síndrome vertiginoso tipo posicional. Además, estos pacientes presentan un mayor depósito de otoconias en los canales semicirculares en comparación a sujetos sanos sin la enfermedad ${ }^{20}$. Veinticuatro por ciento de los pacientes presentó el antecedente de algún trastorno inmunológico siendo lo más frecuente alergias a los medicamentos y rinitis alérgica (Figura 1). Por lo que podría existir mayor frecuencia de este tipo de vértigo en pacientes con patologías autoinmunes y alérgica. En relación con la primera, se ha descrito una relación entre el VPPB y la presencia de autoanticuerpos dirigidos contra la glándula tiroides, por lo cual se propone que los complejos inmunes podrían pasar al oído interno generando 
una estimulación mecánica directa de los receptores laberínticos desencadenando el vértigo. También se ha propuesto que podría corresponder a un síndrome autoinmune multiorgánico. Consecuentemente, Papi y cols sugieren evaluar la presencia de autoanticuerpos dirigidos contra la glándula tiroides en pacientes con VPPB ${ }^{11}$. En relación a lo expuesto previamente, se ha descrito igualmente una función inmunomoduladora de la vitamina $D$, la cual podría tener un impacto sobre la autoinmunidad e inflamación ${ }^{21}$. En el caso de las alergias, se ha observado un incremento del VPPB en pacientes con rinitis alérgica, principalmente en mujeres, sin fisiopatología clara descrita en la literatura22.

Las maniobras de reposición de partículas han demostrado ser el tratamiento más eficiente para el VPPB. Existen diversas maniobras de reposición de partícula dependiendo del CSC afectado, sin embargo, se debe considerar que típicamente el VPPB tiende a presentar remisión espontánea luego de días o semanas posterior a ocurrida su primera manifestación lo que se atribuye al bajo contenido de calcio $(20 \mu \mathrm{M})$ en la endolinfa que puede disolver la otoconia dislocada generando una disminución progresiva de los síntomas ${ }^{23}$. Cuando presentan mayor contenido de calcio en la endolinfa, ésta pierde su capacidad para disolver las otoconias ${ }^{24}$. Dado que la afectación más común es la canalolitiasis del CSC posterior, la maniobra de Epley es considerada la maniobra estándar para su tratamiento ${ }^{25}$. El movimiento de las otoconias dentro del canal está relacionado con los movimientos cefálicos realizados durante la maniobra para lograr reposicionarlas en el utrícul $^{26}$. La maniobra de Semont por su parte, traslada los otolitos hacia el utrículo a través de movimientos bruscos, por lo que es utilizada como una alternativa para casos de cupulolitiasis del CSC posterior ${ }^{27}$. Estas últimas dos son las maniobras más utilizadas dada la alta prevalencia de VPPB en CSC posterior tanto en el mundo como en nuestro país ${ }^{17,28}$.

Además, existen distintas maniobras de reposición en el caso de la afectación de los CSC laterales que dependen de la ubicación de los otolitos en el CSC (brazo posterior, brazo anterior, cupulolitiasis o canalolitiasis). Las más utilizadas para la variante del CSC lateral brazo posterior son las maniobras de Gufoni y Lempert (o maniobra de barbacoa) aunque hoy la más utilizada para esta variable es la maniobra de Gufoni ya que es más fácil de realizar sobre todo en pacientes adultos mayores, obesos, con alteraciones cervicales o musculoesqueléticas ${ }^{29,30}$. En los casos en donde las otoconias se encuentren en el brazo anterior 0 adheridos a la cúpula del CSC lateral las maniobras de reposición más utilizadas son Gufoni-Appiani y la maniobra de Zuma, esta última logra reposicionar las otoconias ubicadas en el brazo anterior del CSC lateral como las que están ubicadas en la cúpula del utrículo, sin necesidad de realizar una segunda maniobra de Gufoni en el caso de transformación geotrópica ${ }^{29,31}$.

Con respecto a la tasa de éxito de las maniobras en esta serie, se obtuvo un éxito total de $77 \%$ con la realización de una sola maniobra en todos los casos. Al desglosar las maniobras, la maniobra de Epley en pacientes con compromiso de los CSC posteriores obtuvo una tasa de éxito de $76 \%$ con la realización de una sola maniobra. Asimismo, la maniobra de Lempert obtuvo la misma tasa de éxito, lo que se correlaciona con la literatura. Se obtuvo una tasa de éxito de $86 \%$ para los casos de canalolitiasis unilateral sin cupulolitiasis. El compromiso de más de un canal y la presencia de cupulolitiasis presentan menores tasas de éxito requiriendo más de una maniobra para la remisión de la sintomatología.

Este estudio es un estudio retrospectivo por lo cual una de las limitaciones es que no siempre existe un registro de todos los datos que se quieren obtener. Además, no se pueden obtener factores de riesgo, solo asociaciones dado que existen factores confundentes asociados que no se aislaron. Por lo tanto, a futuro se deberían realizar estudios prospectivos en población de mayor riesgo con manejo de comorbilidades y factores de riesgo en el tiempo, para poder evidenciar más que asociaciones.

\section{CONCLUSIÓN}

EI VPPB fue más frecuente en mujeres y pacientes con edad promedio de 63 años. Se puede asociar a múltiples comorbilidades como enfermedades cardiovasculares, osteoporosis/osteopenia, y otras 
patologías otológicas que deben ser estudiadas y manejadas correctamente en estos pacientes. La mayoría presentó canalolitiasis unilateral derecha lográndose resolución del cuadro con

\section{BIBLIOGRAFÍA}

1. Kozak HH, Dündar MA, Uca AU, Uguz F, Turgut $K$, Altas M, et AL. Anxiety, mood, and personality disorders in patients with benign paroxysmal positional vertigo. Noropsikiyatri Ars 2018; 55 : 49-53.

2. Carnevale $C$, Arancibia-Tagle DJ, Rizzo-Riera E, TilPerez G, Sarría-Echegaray PL, Rama-Lopez JJ, et al. Efficacy of particle repositioning manoeuvres in benign positional paroxysmal vertigo: A revision of 176 cases treated in a tertiary care centre. Acta Otorrinolaringol Esp 2018; 69: 201-7.

3. Chan KC, Tsal YT, Yao-Hsu Y, Chen PC. Osteoporosis is associated with increased risk for benign paroxsymal vertigo: a nationwide populationbased study. Arch Osteoporos 2017; 1: 106.

4. Chu CH, Liu CJ, Lin LY, Chen TJ, Wang SJ. Migraine is associated with an increased risk for benign paroxysmal positional vertigo: a nationwide population-based study. J Headache Pain 2015; 16: 1-7.

5. Cetin YS, Ozmen OA, Demir Ul, Kasapoglu F, Basut 0 , Coskun $H$. Comparison of the effectiveness of brandt-daroff vestibular training and epley canalith repositioning maneuver in benign paroxysmal positional vertigo long term result: A randomized prospective clinical trial. Pakistan J Med Sci 2018; 34: 558-63.

6. Helminski J0, Janssen I, Kotaspouikis D, Kovacs K, Sheldon P, McQueen K, et al. Strategies to prevent recurrence of benign paroxysmal positional vertigo. Arch Otolaryngol Head Neck Surg 2005; 131: 344-8.

7. Luryi AL, Lawrence J, Bojrab DI, LaRouere M, BABU S, ZapPIA J, et al. Recurrence in benign paroxysmal positional vertigo: A large, singleinstitution study. Otol Neurotol 2018; 39: 622-7.

8. Webster G, Sens PM, Salmito MC, Cavalcante JDR, dos Santos PRB, da Silva ALM, et al. Hyperinsulinemia and hyperglycemia: Risk factors for recurrence of benign paroxysmal una maniobra reposición, sobre todo los pacientes con diagnóstico de canalolitiasis del CSC posterior tratados con maniobra de Epley. El VPPB fue más frecuente en primavera y otoño.

positional vertigo. Braz J Otorhinolaryngo/2015; 81: 347-51.

9. Vázquez PP, Gutiérrez VF, Soto- A, Domínguez 0, López-ESCAMEz JA. Guía de práctica clínica para el diagnóstico y tratamiento del vértigo posicional paroxístico benigno. Acta Otorrinolaringol Esp 2018; 69: 345-66.

10. Neira Méndez P, Valenzuela PV, Viada Lozano J. Semiótica del vértigo postural paroxístico benigno. Rev Otorrinolaringol Cir Cabeza Cuello 2001; 61: 5-12.

11. Papi G, Guidetti G, Corsello SM, Donato C Di, Pontecorvi A. The Association Between Benign Paroxysmal Positional Vertigo and Autoimmune Chronic Thyroiditis Is Not Related to Thyroid Status. Thyroid 2010; 20: 237-8.

12. Prim-Espada M, De Diego-Sastre J, Pérez-Fernandez E. Estudio metaanalítico de la eficacia de la maniobra de Epley en el vértigo posicional paroxístico benigno. Neurología 2010; 25: 295-9.

13. Rico-Romero B, Ishiwara-Niembro K, SáncheZ-péreZ Y. Vértigo postural paroxístico benigno del canal semicircular horizontal (VPPB-CSH) Benign paroxysmal positional vertigo of horizontal semicircular canal ( BPPV-HSC ). Rev Mex Comun Audiol Otoneurología y Foniatría 2012; 1: 119-25.

14. Instrum RS, Parnes S. Benign Paroxysmal Positional Vertigo. Adv Otorhinolaryngol 2019; 82: 67-76.

15. RнIм G II. Serum vitamin D and recurrent benign paroxysmal positional vertigo. Laryngoscope Investig Otolaryngol 2016; 1: 150-3.

16. Vestibulares Dlt, Society B, Brevern M Von, Bertholon P, Brandt T, Fife T, et al. Vértigo posicional paroxístico benigno: criterios diagnósticos. Documento de consenso del Comité para la Clasificación de los Trastornos Vestibulares de la Bárány Society. Acta Otorrinolaringol Esp 2017; 68: 349-60. http:// dx.doi.org/10.1016/j.otorri.2017.02.007.

17. Riveros H, Anabalón J, Correa C. Resultados de la nueva maniobra de reposición de partículas para 
el tratamiento del vértigo postural paroxístico benigno. Rev Otorrinolaringol Cir Cabeza Cuello 2004; 64: 51-6.

18. JaRa V, Paccot M. Implementación del enfoque de riesgo en el programa de salud cardiovascular. Minsal, 2017.

19. Meghus S, Murphy D, Nunney I, Phillips JS. The Seasonal Variation of Benign Paroxysmal Positional Vertigo. Otol Neurotol 2017; 38: 1315-8.

20. Luryi AL, Lawrence J, Bojrab D, LaRouere M, Babu S, Hong R, et AL. Patient, disease, and outcome characteristics of benign paroxysmal positional vertigo with and without Meniere's disease. Acta Otolaryngol 2018; 0??: 1-5.

21. BüKI $B$, Jünger $H, Z$ Zhang $Y$ LY. The Price of Immune Responses and the Role of Vitamin D in the Inner Ear. Otol Neurotol AÑO?; 40: 701-9.

22. Lundberg YW, Ogun OA, Janky KL, Cohn ES, Bu B. Gender-Based Comorbidity in Benign Paroxysmal Positional Vertigo. PLoS One 2014; 9(9): 1-9.

23. Imal T, Ito M, Takeda N, Uno A, Matsunaga T, Sekine $\mathrm{K}, \mathrm{ET}$ AL. Natural course of vertigo in patients with benign paroxysmal positional. Neurology 2005; 64: 920-1.

24. Zucca G, Valli S, Valli P, Perin P, Mira E. Why do benign paroxysmal positional vertigo episodes recover spontaneouslu? Criteria for the Evaluation of Otoconia Dissolution Rate. $J$ Vestib Res 1998; 8: 325-9.
25. Guneri E, Kustutan 0. The Effects of Betahistine in Addition to Epley Maneuver in Posterior Canal Benign Paroxysmal Positional Vertigo. Otolaryngol Head Neck Surg 2012; 146: 104-8.

26. EPLEY JM. The canalith repositioning procedure : For treatment of benign paroxysmal positional vertigo. Otolaryngol Head Neck Surg 1992; 107 : 399-405.

27. Semont A, Freyssb G, Vitte E. Curing the BPPV with a Liberatory Maneuver. Adv Otorhinolaryngol 1988; 42: 290-3.

28. Prokopakis E, Vlastos im, Tsagournisakis M, Christodoulou P, Kawauchi H, Velegrakis G. Canalith Repositioning Procedures among 965 Patients with Benign Paroxysmal Positional Vertigo. Audiol Neurootol 2013; 18: 83-8.

29. Appiani GC, Catania G, Gagliardi M, Cuiuli G. Repositioning Maneuver for the Treatment of the Apogeotropic Variant of Horizontal Canal Benign Paroxysmal Positional Vertigo. Otol Neurotol 2005; 26: 257-60.

30. Oron Y, Cohen-Atsmoni S, Len A, Roth Y. Treatment of Horizontal Canal BPPV : Pathophysiology, Available Maneuvers, and Recommended Treatment. Laryngoscope 2015; 125: 1959-64.

31. Zuma F, Maia C. New treatment strategy for apogeotropic horizontal canal benign paroxysmal positional vertigo. Audiol Res 2016; 6: 36-9. 